\title{
UMTS Release 99/4 Airlink Enhancement for supporting MBMS Services
}

\author{
M.Chuah*1 \\ chuah@cse.lehigh.edu \\ Lehigh University \\ Bethlehem, PA18015
}

Teck Hu
calhu@lucent.com
Lucent Technologies
Whippany, NJ

W. Luo*1

weiluo@broadcom.com

Broadcom Inc.

Matawan, NJ

${ }^{1}$ in 3GPP. Then, in Section 3, we describe a few options for supporting MBMS services using UMTS Release 99/4 airlink channels. We discuss the possibility of using DSCH as well as FACH channels. We discuss the advantages/disadvantages of using such channels and what enhancements need to be made to deliver MBMS traffic efficiently (in terms of power requirement). In Section 4, we present some simulation results of different combinations of the options discussed in Section 3. We conclude in Section 5.

\section{PROPOSED UMTS MBMS ARCHITECTURE} multicast as well as dedicated channels to serve all MBMS users. Some users who are in bad conditions are served using dedicated channels and those who are in relatively good conditions are served using a multicast channel. Last but not least, we present simulation results to give us an idea of how much power is required to support MBMS.

Keywords: Multimedia Broadcast Multicast Services, airlink enhancement for multicast

\section{INTRODUCTION}

Along with the widespread deployment of wireless networks, the fast-improving capabilities of mobile devices, and an increasingly sophisticated mobile work force worldwide, content and service providers are increasingly interested in supporting multicast communications over wireless networks. Many new e-services can be made available if multicast wireless services are available e.g. distance education and entertainment services. Such applications require high bandwidth and near-real-time wireless multicast for quality viewing. In addition, important tactical information may be multicast to users, tanks, and planes in emergency situations or battlefield environments. Such applications demand secure and reliable wireless multicast services with minimal delay. Thus, supporting multicast feature over wireless networks is an important and challenging goal, but several issues must be addressed before group applications can be deployed on a large scale over wireless networks. Recently, work has begun in both $3 \mathrm{GPP}$ and $3 \mathrm{GPP} 2$ to enhance $3 \mathrm{G}$ networks to support multimedia multicast/broadcast services. $3 \mathrm{G}$ networks are designed to provide higher airlink bandwidth and better QoS support than the second generation cellular networks and hence can provide better environments for supporting multimedia multicast/broadcast services.

In this paper, we describe several proposals on how UMTS R99 airlink channels can be enhanced to support multimedia broadcast/multicast services. In Section 2, we give a brief description of the MBMS architecture defined

\footnotetext{
1 The work was done when both authors were with Bell Laboratories
} Service Center is provided to house the MBMS data as well as to distribute layer 3 MBMS content availability information to all subscribers.. The MBMS data source can be located within the BM-SC or in a separate box. In this architecture, Serving Gateway Support Node (SGSN) performs control functions for individual users and consolidates all individual users of the same MBMS service into one single MBMS service. There is only one MBMSGTP tunnel between each SGSN and GGSN for each single MBMS service. GGSN terminates MBMS GTP tunnels and links such tunnels via IP multicast [1] with the multicast data source. There is another alternative for delivering multicast data. If SGSNs also understand IP multicast, then GGSN can send multicast data using special IP multicast address (just for the UMTS network) and all SGSNs that need to receive this data can then subscribe to this special IP multicast address.

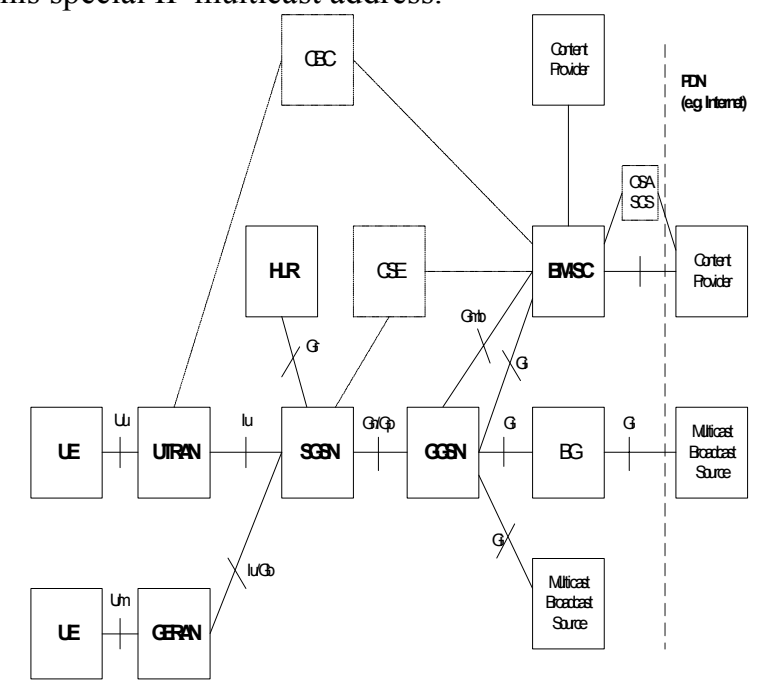


Figure 1: 3GPP Reference architecture for MBMS [2]

Some changes are required in the signalling and bearer setup procedures between a User Equipment (UE) and an Radio Network Controller (RNC) to enable MBMS services. For an example, UE needs to register with RNC for MBMS services. RNC needs to register with SGSN if it does not have the MBMS data stream that the UE is interested in yet. When UE moves from one base station to another, mobility management scheme needs to be enhanced to ensure that the reception of the MBMS stream that the UE subscribed to will not be interrupted. More details can be found in [2].

\section{RELEASE 99/4 AIRLINK ENHANCEMENTS FOR MBMS}

In the interference-limited CDMA system, the downlink capacity is limited by the base station transmission power. The point-to-multipoint communication nature of MBMS requires higher base station transmission power than the unicast service.

Because of the intrinsic complexity associated with the multipoint-to-point feedback, the MBMS traffic is delivered using unacknowledged mode transmission, which means there is no retransmission of the lost data blocks. The only available error control scheme is through channel coding. The channel without retransmission is much less tolerant to the block errors than the channel with retransmission. Therefore, the MBMS service must have a lower block error rate target than the unicast service for the same application. This translates into higher target signal-tointerference ratio (SIR) requirement and higher transmission power.

The MBMS service typically requires that all the MBMS group members in a cell can receive the service. Therefore, the required MBMS transmission power is determined based on the user who has the highest path loss to the base station. Statistically, to maintain a reliable communication towards multiple users requires higher transmission power than towards a single user if the users are uniformly distributed within a cell. In addition, if power control is used, the transmission power has to be adapted to the user who suffers the highest instantaneous path loss to the base station.

In UMTS release 99, two types of common transport channels [6] are defined to support point-to-multipoint communication: Forward Access Channel (FACH) and Downlink Shared Channel (DSCH). Dedicated Channel (DCH) is normally used to deliver unicast data. When the number of MBMS users in a cell is small, it is possible to use one DCH for each MBMS user and the total transmission power required may still be lower than the required power if $\mathrm{FACH}$ would be used. The advantage of DCH over FACH is that DCH is inner-loop and outer-loop power controlled. The disadvantage of DCH is that the radio resource of each channel is dedicated to each user. The transmission power and spreading codes cannot be shared by multiple users even if the data carried on each of the dedicated channel is identical. Such usage of DCH to deliver MBMS data is not suitable for large MBMS group size.

\section{A. Option 1: Using FACH}

FACH does not require any associated uplink channel. The MBMS group member simply needs to tune to the FACH channel to receive the MBMS data traffic without the base station's awareness. The main drawback of FACH is that FACH does not have an uplink channel to report the channel quality, and thus power control is not possible. It is well known that downlink power control brings significant capacity gain to a CDMA system in the fading channel. In addition, if the MBMS group members' SIR is not known at the time when the MBMS service starts, then, the transmission power of the FACH has to be sufficiently high to cover the whole cell. For example, to cover one of the three 120 degree clover-leaf sectors of a cell, a $64 \mathrm{kbps}$ MBMS service carried on FACH will need an Ec/Ior of $0.6 \mathrm{~dB}$ or $87 \%$ of the total sector transmission power under the 3GPP Case 2 channel. This essentially means that MBMS is not feasible unless some power saving techniques are used.

\section{1) Dynamic Power Setting}

Statically setting the base station transmission power to cover the whole cell is wasteful if not even one MBMS user is close to the cell boundary. If the MBMS users' path loss can be monitored, the transmission power of the FACH can be determined based on the worst user's path loss. Thus, the FACH transmission power does not need to cover the whole cell. This is called dynamic power setting [8][9]. As illustrated in Figure, the signal coverage area of dynamic power setting is smaller than static power setting without losing coverage of the MBMS users.

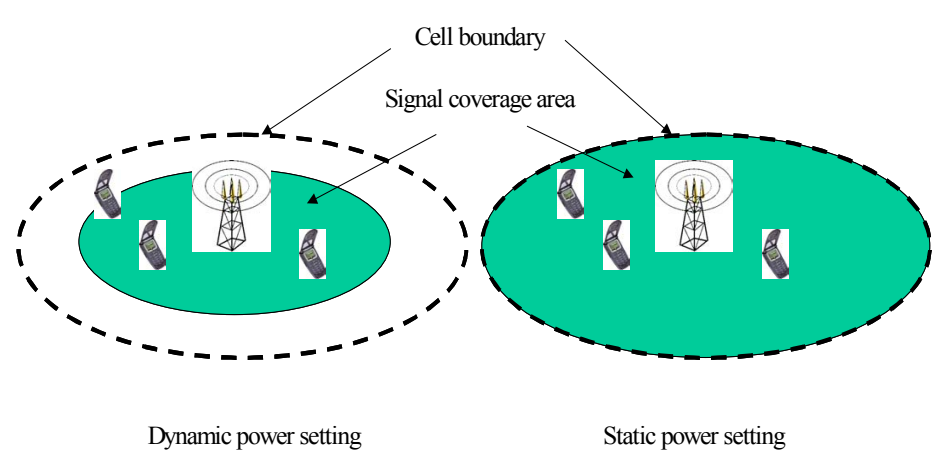

Figure 2. Dynamic power setting vs. static power setting.

To perform dynamic power setting, the MBMS users need to turn on measurement report mechanism while they are on 
the Cell_FACH state. The measurement report rates can be reduced by setting a threshold such that the MBMS users only report when there is a significant change in the measured signal strength. Based on such measurement reports, the base station can adjust the transmission power of the FACH channel to cover say $90 \%$ of the users.

\section{B. Option 2: Using DSCH}

If we desire a closed loop power controlled channel for MBMS service, then DSCH can be used. The DSCH combines the merits of both FACH and DCH. It is power controlled and can be shared by multiple users. The multiple MBMS users however, can share only the PDSCH. To enable power control, each MBMS user needs its own DPCCH channel both in the uplink and downlink. The associated DPCCH overhead increases as the number of MBMS users increases.

The multicast power control of PDSCH is another challenging problem. The most obvious solution is to do power control based on the instantaneous worst user. That is, the DSCH transmission power is reduced only when all MBMS users in the sector command the power to be decreased; the PDSCH transmission power is increased when at least one MBMS user in the sector commands transmission power to be increased. So, the PDSCH transmission power is the maximum of all users' required transmission power. For each individual user, however, the transmission power is often higher than its required power. This implies that the user perceives unnecessarily better than its required radio quality at the cost of increased average PDSCH transmission power. This problem becomes more serious as the MBMS group size increases. In Figure 3 [11], the worst user based $\mathrm{PDSCH}$ power control is compared with the solution using FACH with dynamic power setting. The simulation assumptions used to generate Figure 3 are listed in Table 1.

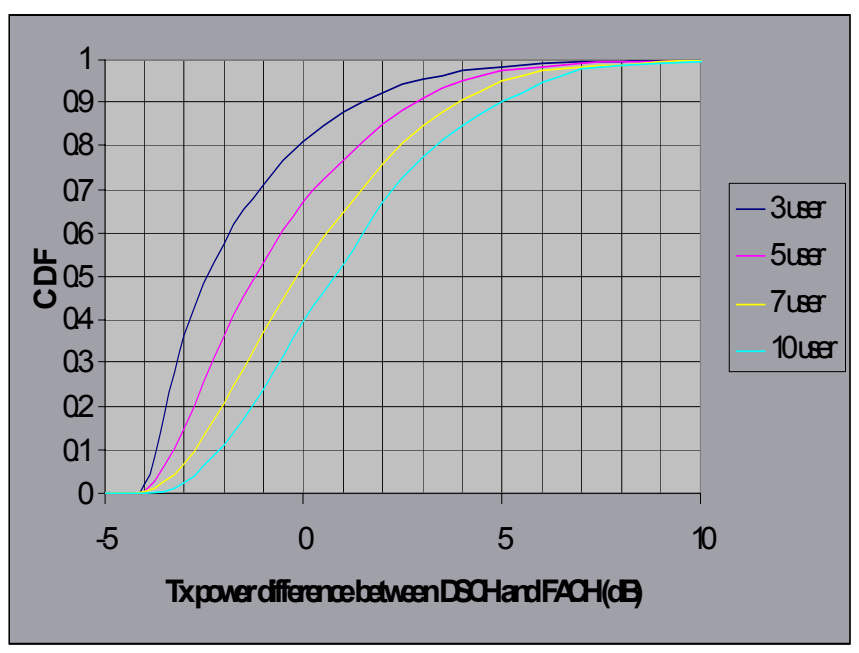

Figure 3. Power Controlled DSCH vs FACH with Dynamic Power Setting

\begin{tabular}{|l|c|}
\hline Parameters & Assumptions \\
\hline Cell shape & Clover leaf \\
\hline Cell radius & $2 \mathrm{~km}$ \\
\hline Sectors/cell & 3 \\
\hline Antenna gain & $-174 \mathrm{dBi}$ \\
\hline $\begin{array}{l}\text { No (Noise spectrum } \\
\text { density) }\end{array}$ & $7 \mathrm{~dB}$ \\
\hline $\begin{array}{l}\text { Log-normal shadow } \\
\text { fading standard deviation }\end{array}$ & $20 \mathrm{~W}$ \\
\hline $\begin{array}{l}\text { Maximum base station } \\
\text { transmit power }\end{array}$ & $0 \mathrm{dBi}$ \\
\hline UE antenna gain & $3 \mathrm{~km} / \mathrm{h}$ \\
\hline Mobile speed & $64 \mathrm{~kb} / \mathrm{s}$ \\
\hline Data rate & \\
\hline
\end{tabular}

Table 1: Simulation Assumptions.

Note that the overhead of the associated uplink and downlink DPCCH channel is not included in this analysis. If that is taken into account, the DSCH power consumption number will be larger. We also assume that for a single user link, the power control results in a $4 \mathrm{~dB}$ gain. The advantage of using DSCH diminishes as the number of users increases. For an example, when the number of users is three, with $50 \%$ probability DSCH consumes about $2.5 \mathrm{~dB}$ less power than $\mathrm{FACH}$, and with $80 \%$ probability $\mathrm{DSCH}$ consumes less power than FACH. When the number of users is 10 , with $50 \%$ probability DSCH consumes $1 \mathrm{~dB}$ more power than $\mathrm{FACH}$. We observe that the $\mathrm{DSCH}$ solution consumes more power than the solution using FACH with dynamic power setting based on the worst user's condition The above analysis demonstrates that the worst user based DSCH power control is not suitable for MBMS. Further study on more effective power control algorithm is warranted. We will not discuss Option 2 further for the rest of the paper.

\section{Option 3: Using longer TTI and Space Diversity}

Two methods can be employed in the physical layer to benefit every member of the MBMS group in a cell.

- Longer Transmission Time Interval to exploit the time diversity

- Space Diversity Transmission to exploit the space diversity

In recent MBMS standard meetings, STTD has been proposed to enhance the performance of $\mathrm{FACH}$ channel for delivering 64kbps MBMS traffic. The MBMS traffic is not interactive, therefore it is relatively tolerant to the transmission delay. It is well-known that longer interleaving depth can counteract the fading effects. In Release 99/4, TTI can take certain discrete values: $\{10 \mathrm{~ms}, 20 \mathrm{~ms}, 40 \mathrm{~ms}$, 
$80 \mathrm{~ms}$ \}, where $20 \mathrm{~ms}$ is the default value for a $\mathrm{FACH}$ channel. It has been shown [7] that increasing Transmission Time Interval (TTI) length from $20 \mathrm{~ms}$ to $80 \mathrm{~ms}$ without increasing the coding rate can provide $2-4 \mathrm{~dB}$ gain in a typical urban pedestrian fading environment. The use of longer TTI introduces more complexity and larger memory space requirement in the mobile station.

\section{Option 4: Rate Splitting [8,9]}

If the MBMS data stream is scalable [12], it can be split into several streams with different importance. Only the most important stream is sent to all the users in the cell to provide the basic service. The less important or enhancement streams are sent with less amount of power or coding protection. Only the users who have better channel conditions can receive the enhancement data stream. Thus, the transmission power for the most important MBMS stream can be reduced due to a lower data rate, and the transmission power for the enhancement streams can also be reduced because the coverage requirement is relaxed. This is called rate splitting.

\section{E. Option 5: Multiple DCH channels + FACH [10]}

In this approach, one can dynamically decide on a value $\mathrm{K}$ such that $\mathrm{K}$ users will be served using a broadcast FACH channel while (M-K) users will be served using point-topoint links and that the total power required to support such a combination of channels will be less than just using a broadcast $\mathrm{FACH}$ channel to reach all $\mathrm{M}$ users. The transmission power used for the broadcast FACH channel will be based on the worst-case user among that $\mathrm{K}$ users. This approach is appealing because when a small number of users are in bad position, we need not transmit large power for the broadcast FACH channel, and by using DCH with the soft handoff feature, the power required to reach the bad users can be reduced.

One can also combine the different options listed above. For an example, one can use Option 1 with dynamic setting together with Option 4, another possibility is to implement a combination of Option 1, $3 \& 4$.

\section{SimULATION Results}

In this section, we present some simulation results of the various options and compare these different options. The results for Option 5 will be presented in a longer version of the paper [14]. The basic assumptions used for such simulations are the same as in Section 3 and are listed in Table 1. The link level simulation results of $64 \mathrm{Kbps}$ under Case $23 \mathrm{~km} / \mathrm{h}$ channel that we use are shown in Table 2.

Table 2: Required Ec/Ior for $64 \mathrm{Kbps}, 3 \mathrm{~km} / \mathrm{h}$ Case 2

\begin{tabular}{|l|l|l|l|l|}
\hline $\operatorname{lor} / \operatorname{loc}(\mathrm{dB})$ & -6 & -1 & 4 & 9 \\
\hline
\end{tabular}

\begin{tabular}{|l|l|l|l|l|}
\hline Ec/lor (dB) & -0.6 & -6 & -10.7 & -14.5 \\
\hline
\end{tabular}

\section{A. Options 1 \& 4: FACH with static/dynamic power setting $[8,9]$}

The comparison of the single-rate and dual-rate schemes with static power setting is shown In Figure 4. The curve of "Single Rate" shows that the requirement of Ec/Ior decreases with the coverage percentage. It can be seen that for $99 \%$ cell coverage, about $-1.7 \mathrm{~dB}$ of Ec/Ior is needed for single-rate scheme. The curve of "Dual rate" shows the requirement of Ec/Ior of the dual-rate scheme. The coverage percentage of the first stream is always close to $100 \%$ so that every user in the cell can receive the basic service and the coverage percentage of the second stream is the variable whose value is shown in the $\mathrm{x}$-axis. We see that to cover $50 \%$ of the cell with the enhancement stream, about $-4 \mathrm{~dB}$ of $\mathrm{Ec} /$ Ior is required which is $2.2 \mathrm{~dB}$ less than the single-rate scheme. The power saving of the dual-rate scheme decreases with the increasing cell coverage of the enhancement stream. Interested users can refer to [9] for the results with dynamic power setting.

\section{B. Options 1 (static power setting), $3 \& 4$ [13]}

In this set of simulations, the dynamic power setting feature for Option 1 is disabled. The value of the transmission power is assumed to be static and is set to cover certain percentage of the geographical locations within a cell. Two possible MBMS schemes can be used. The first one is to use a single rate stream, which is carried on a single $64 \mathrm{kbps}$ channel and sent to the covered areas. The second one is to use two data streams, each of $32 \mathrm{kbps}$. The first stream carries the most important data and is used to provide the basic quality of service. The second stream carries the enhancement data and is used to provide the enhanced quality of service, with the full $64 \mathrm{kbps}$. The first stream is sent to the whole area in the cell. The second stream is sent only to cover certain percentage of the cell.

The performance of dual rate splitting is compared with single stream FACH for TTI of $20 \mathrm{~ms}, 80 \mathrm{~ms}, 160 \mathrm{~ms}$, and $320 \mathrm{~ms}$. For $99 \%$ cell coverage, about $-1.68 \mathrm{~dB},-5.66 \mathrm{~dB}$, $7.27 \mathrm{~dB},-7.93 \mathrm{~dB}$, and $-8.7 \mathrm{~dB}$ of Ec/Ior are needed for TTI of $10 \mathrm{~ms}, 20 \mathrm{~ms}, 80 \mathrm{~ms}, 160 \mathrm{~ms}$, and $320 \mathrm{~ms}$ respectively for the single-rate scheme. Rate splitting with dual rate, as shown in Figure 5, requires about $-4.0 \mathrm{~dB},-7.8 \mathrm{~dB},-9.3 \mathrm{~dB}$, $10 \mathrm{~dB}$, and $-10.7 \mathrm{~dB}$ for TTI of $10 \mathrm{~ms}, 20 \mathrm{~ms}, 80 \mathrm{~ms}, 160 \mathrm{~ms}$, and $320 \mathrm{~ms}$ respectively for a second stream cell coverage of $50 \%$. This yields an improvement in the range of $\sim 2.0 \mathrm{~dB}$ over the single rate scheme. As a result, dual rate splitting requires total Ec/Ior of $40 \%$ and $8.5 \%$ for TTI of $10 \mathrm{~ms}$ and $320 \mathrm{~ms}$ respectively compared to $68 \%$ and $13.5 \%$ of total $\mathrm{Ec} / \mathrm{Ior}$ for single rate $\mathrm{FACH}$ at $64 \mathrm{kbps}$. 


\section{CONCLUDing REMARKS}

In this paper, we first describe the 3GPP architecture for MBMS. Then, we present several options for carrying MBMS traffic over the UMTS Release 99/4 airlink

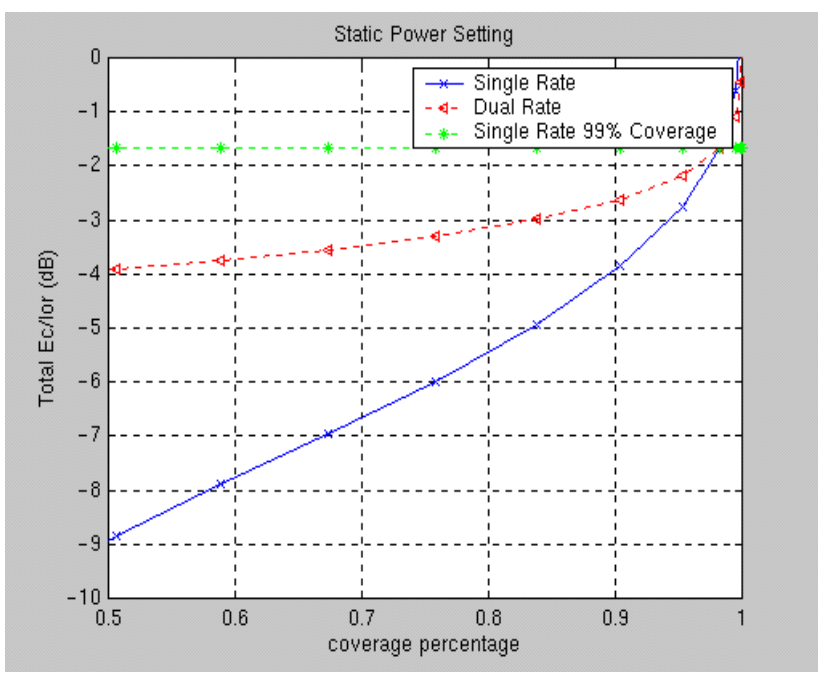

Figure 4. Static Power Setting

channels. We discuss the pros/cons of different options. We discuss why DSCH with a power control scheme that is based on worst case user's condition is not a viable solution. We also discuss how rate splitting, long TTI and STTD can be used to reduce the power requirement of using $\mathrm{FACH}$ as the multicast channel for MBMS services. We also present some simulation results on a combination of different options. Our preliminary results indicate that the combinations of Option 1 (static power setting), $3 \& 4$ as well as Option 5 seem to be the two most promising options for supporting MBMS in UMTS Release 99/4 systems. More work needs to be done in the area of mobility management and signalling procedures (e.g. paging idle users to notify them of the availability of MBMS data streams) before MBMS can be deployed.

\section{REFERENCES}

[1] S. Deering and D. Cheriton, "Multicast routing in datagram inter-networks and extended LANs", ACM Transactions on Computer Systems , 8(2): 85110, May, 1990

[2] 3GPP TS 23.240 V.0.5.0, "MBMS Architecture and Functional Description", April, 2003

[3] 3GPP TSG-RAN WG1\#28, "Power allocation for MBMS”, Ericsson, R1-021224, October, 2002

[4] S. M. Alamouti, "A Simple Transmit Diversity Technique for Wireless Communications", IEEE JSAC, Vol 16, Oct 1998 pp 1451-58.

[5] S. Bäro, G. Bauch, and A. Hansmann, "Improved Codes for Space-time Trellis-coded Modulation,",
IEEE Communication. Letters., vol. 4, Jan. 2000, pp. $20-2$.

[6] 3GPP TS 25.211 V3.11.0 (2002-06),"Physical channels and mapping of transport channels onto physical channels (FDD)" (Release 1999).

[7] 3GPP TSG-RAN WG1\#28, "Evaluation of combining gains for MBMS (incl. STTD)," Qualcomm, R1-021234, October, 2002.

[8] 3GPP TSG-RAN WG1\#28, "MBMS Power Usage," Lucent Technologies, R1-021239, October, 2002

[9] 3GPP TSG-RAN WG1\#29, "Power Control for FACH," Lucent Technologies, R1-021324, November 2002.

[10] 3GPP TSG-RAN WG1\#28, "Power Usage for Mixed FACH and DCH for MBMS," Lucent Technologies, R1-021240, October, 2002.

[11] 3GPP TSG-RAN, WG1\#29, "Comparison of DSCH and FACH for MBMS," Lucent Technologies, R1021325, November 2002.

[12] P. Bender, P. Black, M. Grob, R. Padovani, N.Sindhushyana, S. Viterbi, "CDMA/HDR: a bandwidth efficient high speed wireless data service for nomadic users", IEEE Communications Magazine, Vol. 38, pp. 70-77, July, 2000

[13] 3GPP TSG-RAN, WG1\#33, "Rate Splitting for Scalable MBMS," Lucent Technologies, R1-030778, August 2003.

[14] M. Chuah, T. Hu, W. Luo, UMTS Release 99/4 Airlink Enhancement for supporting MBMS Services, to be submitted to IEEE transactions on wireless communications.

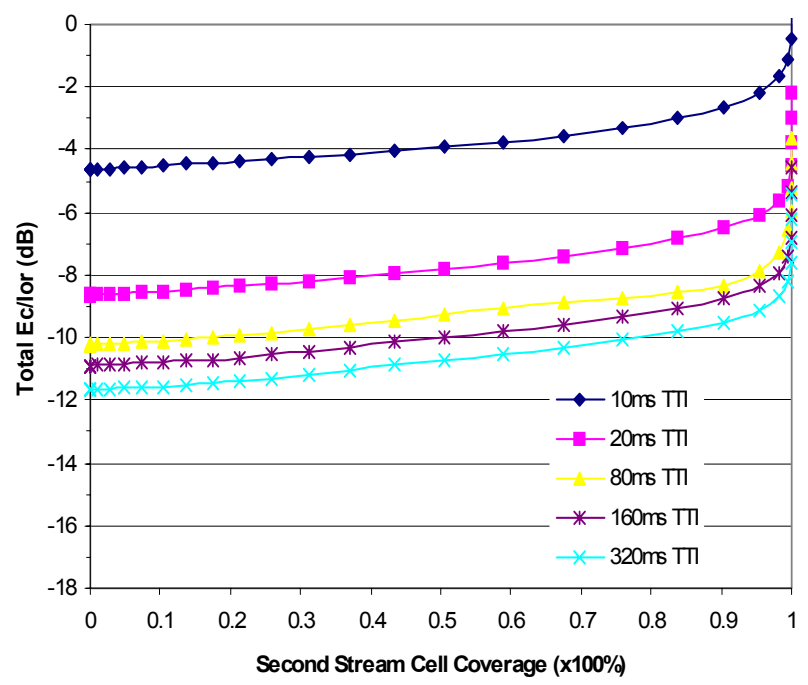

Figure 5. Dual Rate FACH 64kbps 